\title{
Ansiedade e Depressão Materna e Relatos sobre o Bebê Prematuro ${ }^{1}$
}

\author{
Ingrid Duarte Pinto \\ Flávia Helena Pereira Padovani \\ Maria Beatriz Martins Linhares ${ }^{2}$ \\ Universidade de São Paulo, Ribeirão Preto
}

\begin{abstract}
RESUMO - Os objetivos do presente estudo foram: (a) comparar dois grupos de mães, diferenciados pela presença de sintomas emocionais clínicos de ansiedade e depressão, quanto aos relatos sobre o seu bebê prematuro; (b) verificar relações entre os relatos maternos e características das mães, a história de saúde neonatal do bebê e eventos estressores ambientais. A amostra foi composta por 60 mães, distribuídas em dois grupos: 30 mães com indicadores clínicos (MCIE) e 30 mães sem tais indicadores (MSIE). As mães foram entrevistadas e avaliadas por meio dos seguintes instrumentos: SCID-NP, IDATE e BDI. O prontuário médico também foi analisado. Em ambos os grupos, as mães verbalizaram predominantemente sobre expectativas e concepções positivas acerca do bebê. No entanto, o MCIE relatou mais reações e sentimentos negativos do que o MSIE. Menor peso ao nascimento, menor idade gestacional e maior tempo de internação do bebê na UTI-Neonatal associaram-se com expectativas, reações e sentimentos maternos negativos.
\end{abstract}

Palavras-chave: bebê pré-termo; ansiedade; depressão; relato verbal.

\section{Maternal Anxiety and Depression and Reports about the Premature Infant}

\begin{abstract}
The aims of the present study were: (a) to compare two groups of mothers differentiated by the presence of clinical emotional symptoms of anxiety and depression, in respect of verbal reports about their premature infants; (b) to verify the relation between verbal reports and variables of mothers' characteristics, infants' neonatal health history, and distressful life events. The sample was composed by 60 mothers, allocated into two groups: 30 with emotional clinical symptoms (ECSM) and 30 with no symptoms (NCSM). The mothers were assessed by SCID-NP, STAI, and BDI and were interviewed by an interview-guide. The medical chart was also reviewed. Both groups reported predominantly positive expectations and concepts about the babies. Otherwise, MCES reported more negative reactions and feelings than MSES. Lower birthweight, lower gestational age and higher length-stay NICU hospitalization were associated with negative expectations, reactions, and feelings of the mothers.
\end{abstract}

Keywords: preterm infants; anxiety; depression; verbal report.

As mães de bebês prematuros internados em Unidade de Terapia Intensiva Neonatal (UTIN) apresentam escores indicativos de sintomas clínicos de ansiedade, disforia e/ ou depressão (Correia \& Linhares, 2007; Davis, Edwards, Mohay \& Wollin, 2003; Doering, Moser \& Dracup, 2000; Padovani, Linhares, Carvalho, Duarte \& Martinez, 2004; Zanardo \& Freato, 2001). Dessa forma, essas mães apresentam alto risco para problemas de saúde mental, tornando-se mais fragilizadas para o enfrentamento adequado do nascimento prematuro e menos adaptadas aos cuidados iniciais do bebê (Kennell \& Klaus, 1998; Klaus \& Kennell, 1992/1993; Linhares, Carvalho, Correia, Gaspardo \& Padovani, 2006).

Estudos sobre aspectos emocionais maternos visam (1) avaliar indicadores de ansiedade e depressão, por meio de instrumentos psicométricos (Davis \& cols., 2003; Doering \& cols., 2000; Feldman, 2007; Rice, Jones \& Thapar, 2007; Zanardo \& Freato, 2001), ou (2) compreender os aspectos subjetivos experimentados pelas mães no enfrentamento do

1 Apoio financeiro: CNPq, FAPESP.

2 Endereç: Laboratório de Prevenção de Problemas do Desenvolvimento e Comportamento de Crianças (salas 52/53), Prédio da Saúde Mental da Faculdade de Medicina de Ribeirão Preto - USP. Avenida Tenente Catão Roxo, 2650. Campus Universitário - Monte Alegre. Ribeirão Preto, SP. CEP 14048900. E-mail: linhares@fmrp.usp.br. problema da prematuridade do filho e condições associadas, por meio de entrevistas e análise qualitativa de relatos (Correia, Carvalho \& Linhares, 2008; Flacking, Ewald \& Starrin, 2007; Glaser, Bucher, Moergeli, Fauchère \& Buechi, 2007; Javorski, Caetano, Vasconcelos, Leite \& Scochi, 2004; Roller, 2005).

Nos estudos que utilizam escalas de avaliação do estado emocional materno, destaca-se o uso do Inventário de Ansiedade Traço/Estado - IDATE, para avaliação de ansiedade sob ambos os aspectos de traço-estável e de estado-situacional transitório, e o Inventário Beck - BDI, para avaliação de disforia e depressão (Feldman, 2007; Padovani \& cols., 2004). A ansiedade do tipo estado, que pode ser experimentada por mães de bebês prematuros, trata-se de estado emocional marcado por sentimentos desagradáveis de tensão e apreensão reativos à situação de nascimento e internação do bebê na UTIN (Padovani \& cols., 2004).

Paralelamente à grande contribuição advinda da avaliação de indicadores de ansiedade e depressão materna, Aywarld (2002) destaca que também há necessidade de identificar, por meio da expressão verbal, os sentimentos, medos e ansiedades e dificuldades no enfrentamento do nascimento prematuro do filho. A fragilidade emocional de mães de bebês prematuros pode ser identificada por meio desses relatos so- 
bre a sua experiência, revelando os seguintes aspectos: medo das possíveis consequências do parto prematuro, temor pela saúde do bebê e tristeza por vivenciar uma situação diferente daquela vivenciada por mães de filhos nascidos a termo, as quais podiam acariciá-lo, amamentá-lo e envolvê-lo nos braços a qualquer momento (Monteiro, Silva \& Silva, 2002).

Essa amplitude de preocupações e sentimentos traduz o universo psicológico das mães dos prematuros, no qual pode também coexistir sentimentos de esperança e expectativas positivas. Em um estudo de Linhares e cols. (2006), as mães de bebês prematuros internados em UTIN expressaram em grupos de apoio psicológico, em ordem de maior ocorrência, preocupações com a sobrevivência do bebê e a separação do mesmo, impotência de desempenhar o papel ativo de mãe, alívio e esperança em relação à sobrevivência, desejo de alta hospitalar, culpa relacionada a condições adversas antecedentes ao nascimento, problemas pessoais, alívio quanto às condições de saúde e desenvolvimento e frustrações de suas expectativas relação ao bebê.

Verifica-se, no entanto, que são raros os estudos com o objetivo de analisar em conjunto os indicadores de ansiedade e depressão materna, mensurados objetivamente por avaliação psicológica, e os relatos maternos sobre o impacto emocional do nascimento prematuro do filho, analisados na perspectiva da experiência subjetiva. Além disso, há uma lacuna na literatura relativa à análise da relação entre a experiência materna associada com os indicadores emocionais e as condições de saúde neonatal do bebê, as características pessoais maternas e os indicadores do contexto ambiental.

O presente estudo visa, portanto, atender à questão de analisar as concepções e expectativas relatadas por mães de bebês prematuros levando-se em conta a avaliação psicológica do seu estado emocional e características do bebê e do ambiente. Para tanto, foram definidos os seguintes objetivos: (a) comparar dois grupos de mães, diferenciados pela presença de sintomas emocionais clínicos de ansiedade e depressão, quanto aos relatos sobre o seu bebê prematuro; (b) verificar as relações entre os relatos maternos e as características pessoais das mães, as condições de nascimento e evolução clínica dos bebês e os eventos ambientais estressores.

\section{Método}

\section{Participantes}

A amostra do presente estudo foi composta por 60 mães de bebês pré-termo ( $<37$ semanas de idade gestacional) com muito baixo peso $(\leq 1500 \mathrm{~g})$, internados na UTI-Neonatal (UTIN) ou no Berçário de Médio Risco do Hospital das Clínicas da Faculdade de Medicina de Ribeirão Preto (HC/ FMRP-USP), no período de 2001 a 2003. As mães foram distribuídas em dois grupos, a saber: Grupo MCIE, constituído por 30 mães com indicadores emocionais clínicos de ansiedade e/ ou depressão, e Grupo MSIE, constituído por 30 mães sem esses indicadores.

Essa amostra foi extraída de uma amostra inicial de 70 mães que tiveram bebês pré-termo durante o período de coleta de dados. Foram usados os seguintes critérios de exclusão: mães analfabetas $(n=6)$, mães portadoras do vírus HIV $(n=4)$, considerando que essas variáveis podem influenciar os resultados das avaliações.

O estudo contou com a aprovação do Setor de Neonatologia da Pediatria e do Comitê de Ética em Pesquisa do HC/FMRP - USP. Posteriormente à fase de coleta de dados do presente estudo, as mães receberam apoio psicológico no serviço de Psicologia Pediátrica vinculado à Neonatologia do referido hospital, conforme protocolo de intervenção psicológica descrito por Linhares e cols. (2006).

\section{Instrumentos e Materiais}

Foram utilizados os seguintes instrumentos e materiais: (a) Roteiro de Entrevista (Carvalho, 2005), do qual foram selecionadas questões relativas a idade, nível de escolaridade, número de filhos, ocupação profissional da mãe, situação conjugal e a representação materna acerca do bebê prematuro. Essa temática foi abordada por meio das seguintes questões: "O que você esperava do seu bebê na gravidez?”; "Como você percebe seu bebê agora?"; "O que você espera daqui para frente?"; (b) BDI - Inventário de Depressão de Beck (Cunha, 2001; Gorenstein \& Andrade, 2000); (c) IDATE - Inventário de Ansiedade Traço-Estado (Andrade \& Gorenstein, 2000; Biaggio \& Natalício, 1979); (d) SCID/ Não Paciente - Entrevista Clínica estruturada para DSMIII-R (Del Ben, 1995; Spitzer, Williams, Gibbon \& First, 1989); (e) Escala de Eventos Vitais (Savoia, 2000), que avalia os eventos potencialmente adversos ocorridos nos últimos 12 meses (e.g., morte do cônjuge, separação, dívidas, perda de emprego, doença na família); (f) Prontuários Médicos dos bebês do HC/FMRP-USP, do qual foram extraídos dados sobre a idade gestacional, peso ao nascimento, tempo de internação na UTIN, número de intercorrências durante a internação. Foram obtidos, ainda, os dados para calcular o Índice de Risco Clínico Neonatal (CRIB- Clinical Risk Index for Babies) (Cockburn \& cols., 1993) e classificar a adequação do peso de nascimento em relação à idade gestacional (Marques, 2003), além do Apgar do $5^{\circ}$ minuto. Foram utilizados um gravador e fitas para registro das entrevistas com as mães.

\section{Procedimento}

A coleta de dados foi realizada durante o período de internação do bebê na UTIN e no Berçário de Médio Risco do HC/ FMRP-USP. Após a assinatura do Termo de Consentimento Livre e Esclarecido, foi aplicada a SCID/NP para verificar a presença de antecedentes psiquiátricos. Posteriormente, foram realizadas duas sessões com cada mãe durante o período de internação dos bebês. Na primeira sessão, as mães foram entrevistadas de forma guiada, usando o Roteiro de Entrevista para obter informações gerais sobre a mãe e suas representações acerca do bebê. Em seguida, foi aplicada a Escala de Eventos Vitais para identificar os eventos potencialmente estressores que ocorreram nos últimos 12 meses. Na segunda sessão, foram aplicados os inventários para a avaliação psicológica de ansiedade (IDATE) e depressão (BDI) das 
mães, de forma alternada por participante, a fim de controlar o efeito carry over. Com base nos resultados da avaliação psicológica, as mães foram distribuídas em dois grupos de acordo com a presença de sintomas clínicos de ansiedade e depressão. Realizaram-se consultas aos prontuários médicos dos bebês, para coleta de dados sobre a história e evolução clínica do bebê durante a internação hospitalar.

Para identificar a presença de sintoma clínico de ansiedade foi definida a nota de corte do percentil igual ou maior do que 75 (> quartil 3) no IDATE. Para identificar o sintoma clínico de depressão ou disforia utilizou-se a pontuação acima de 20 e de 15 , respectivamente, como sugerido para pacientes não-diagnosticados (Gorestein \& Andrade, 2000). Os dados do bebê referentes à presença dos fatores de estresse neonatal foram analisados de acordo com o Índice de Risco Neonatal - CRIB (Cockburn \& cols., 1993). Quanto à classificação dos bebês em função da relação entre seu peso de nascimento e a idade gestacional, os bebês foram classificados como: pequenos para a idade gestacional (PIG) e peso adequado para a idade gestacional (AIG) (Marques, 2003). As gravações das entrevistas foram transcritas literalmente e categorizadas de acordo com o sistema de análise desenvolvido por Padovani (2005). O índice de fidedignidade entre dois avaliadores independentes com treino no sistema de análise foi processado utilizando $33 \%$ do total de protocolos. Foi utilizada a seguinte fórmula: (Acordos/Acordos + Desacordos) x 100. O índice de fidedignidade obtido entre os avaliadores foi de $91 \%$.

As categorias e subcategorias do sistema de análise do conteúdo verbal materno utilizadas no presente estudo encontram-se a seguir:

1 - Expectativas relativas ao bebê. 1.1. Positiva: bebê recém-nascido saudável, a termo ou bonito; desejo da alta hospitalar; desenvolvimento, evolução e saúde do bebê no futuro. 1.2. Negativa: problema da prematuridade do bebê. 1.3. Conflituosa: expectativas ambivalentes sobre o bebê. 1.4. Neutra: preferência por gênero do bebê; atribuição de características físicas e comportamentais; 1.5. Ausência de expectativas.

2 - Concepções relativas ao bebê. 2.1. Positiva: desenvolvimento, evolução e saúde do bebê; sentimento de amor e orgulho em relação ao bebê. 2.2. Negativa: fragilidade do bebê nascido prematuro. 2.3. Conflituosa: concepções ambivalentes sobre o bebê. 2.4. Neutra: características físicas e comportamentais do bebê.

3 - Reação e/ou sentimento relativos ao bebê e à internação. 3.1. Positiva: felicidade, alegria, euforia, esperança, confiança, segurança, adaptação e aceitação. 3.2. Negativa: choro, tristeza, raiva, mal-estar, medo, angústia, insegurança, susto, espanto, apreensão, rejeição e revolta. 3.3. Conflituosa: oscilação ou ambivalência de emoções ou sentimentos.

4 - Descrições sobre o bebê: caracterização do padrão de comportamento do bebê; descoberta da gravidez e de situações ocorridas no período de gestação.

5 - Identificação de fatores que influenciam na maternidade. 5.1. Ajuda: características e/ou atitudes do bebê. 5.2. Atrapalha: características e/ou atitudes do bebê.

6 - Outras: expressões de conteúdo apropriado à temática questionada, mas que não se enquadravam nas categorias elaboradas e também não se agrupavam de modo a formar uma nova categoria.
7 - Resíduo: verbalizações que não se enquadravam nas categorias do sistema e que ocorreram em número reduzido.

\section{Análise de dados}

Inicialmente, os dados das avaliações emocionais foram analisados segundo as normas de correção descrita pelos autores das escalas psicométricas. Procedeu-se a análise de estatística descritiva dos dados, em termos de média e desvio padrão ou frequência e proporção, de acordo com a natureza das variáveis contínuas ou discretas. As categorias de conteúdo verbal dos grupos MCIE e MSIE foram quantificadas por meio de frequência e porcentagem. Em seguida, para cada subcategoria foi calculada sua porcentagem dividindo-se a frequência de verbalizações referentes à determinada subcategoria pela frequência total das verbalizações da categoria correspondente em que esta se inseria e multiplicando-se este resultado por 100. A comparação entre grupos (MCIE vs. MSIE) foi realizada por meio do teste não-paramétrico de Mann-Whitney, para variáveis contínuas, ou teste de Quiquadrado, para variáveis categóricas.

Procedeu-se a uma análise específica no grupo MCIE, separando-o em quatro subgrupos, segundo o tipo de indicadores emocionais encontrado na avaliação psicológica das mães. Para comparação entre esses grupos foi utilizado o teste não paramétrico de Kruskal-Wallis, seguido do teste de Mann-Whitney, com correção de Bonferroni.

Foram calculadas as correlações entre as verbalizações maternas e características maternas, características e evolução clínica dos bebês, e eventos ambientais estressores, respectivamente, utilizando-se o teste de correlação de postos de Spearman. Para o tratamento estatístico dos dados utilizou-se o Statistical Package for Social Sciences (SPSS) na versão 12.0. O nível de significância adotado no estudo foi de $5 \%(p \leq 0,05)$.

\section{Resultados}

\section{Caracterização da amostra}

Como era de se esperar, os grupos apresentaram diferenças significativas em relação aos escores de AnsiedadeEstado [MCIE $=58,80( \pm 7,61)$ e MSIE $=41,33( \pm 12,31)$; $p<0,0001]$, Ansiedade-Traço [MCIE $=55,20( \pm 9,44) \mathrm{e}$ MSIE $=40,63( \pm 10,59) ; p<0,0001]$ e Depressão [MCIE $=$ $19,57( \pm 10,98)$ e $\operatorname{MSIE}=6,63( \pm 3,62) ; p<0,0001]$. $\mathrm{O}$ grupo MCIE era composto por 30 mães com diferentes tipos de indicadores emocionais clínicos, que foram classificados em quatro subgrupos, a saber: mães só com ansiedade do tipo estado (MAE, $n=9$ ), mães com indicadores emocionais clínicos combinados de ansiedade-estado e ansiedade-traço ou de ansiedade e depressão (MIC, n=9), mães com antecedentes psiquiátricos (MSCID, n=7) e mães com depressão ou disforia (MDD, $\mathrm{n}=5$ ).

Pode-se verificar, na Tabela 1, que as mães de ambos os grupos eram semelhantes quanto às características de 
idade, escolaridade e situação conjugal. Quanto à ocupação profissional, nota-se que a proporção de mães empregadas era similar entre os grupos, mas houve diferença entre os grupos no que se refere às mães MCIE estarem mais desempregadas do que as mães MSIE; estas eram mais do lar do que empregadas. Além disso, as mães com indicadores emocionais clínicos tinham significativamente mais filhos do que as mães sem indicadores.

Adicionalmente aos resultados da Tabela 1, a análise dos dados sobre os eventos vitais potencialmente estressores do ambiente familiar (desemprego, separação do casal, enfermidade crônica, entre outros) mostrou que não houve diferença estatisticamente significativa entre os grupos [MCIE $=7,48$ $( \pm 2,86)$ e $\mathrm{MSIE}=6,33( \pm 2,64) ; p \leq 0,07]$.

A Tabela 2 indica que as características neonatais dos bebês dos grupos MCIE e MSIE foram semelhantes quanto às seguintes variáveis: sexo, idade gestacional, peso ao nascimento, índice de risco clínico neonatal, Apgar do $5^{\circ}$ minuto, adequação para a idade gestacional e tempo de internação na UTIN. A maioria dos bebês nasceu com idade gestacional próxima a 31 semanas e o peso ao nascimento de ambos os grupos situou-se em torno de 1.100 gramas. A maioria dos bebês era pequena para a idade gestacional nos dois grupos. Os grupos apresentaram alto escore médio de Apgar, situado em torno de 8,6 e a média do índice de risco neonatal, medido por meio do CRIB, esteve próximo a 3 . Esses dados indicam bom prognóstico de evolução clínica dos bebês e baixo índice de risco neonatal para morbi-mortalidade. Deve-se destacar, no entanto, que em ambos os grupos esses dois índices, de Apgar e do CRIB, apresentaram alta amplitude de variação nos escores, o que indica grande variabilidade individual. O tempo de internação na UTIN dos bebês dos dois grupos foi semelhante, situando-se entre 20 a 30 dias, em média, apresentando também alta amplitude de variação.

\section{Relatos maternos sobre o bebê}

Observa-se, na Tabela 3, que as mães de ambos os grupos abordaram, em ordem decrescente de frequência, três grandes temas: expectativas, concepções e reações e/ou sentimentos relativos ao seu bebê. A análise dessas categorias, levandose em conta as suas subcategorias mais frequentes, mostrou que nas expectativas maternas predominaram sobremaneira as de conotação positiva. A expressão de expectativas positivas esteve principalmente relacionada aos aspectos do desenvolvimento, da evolução e da saúde do bebê no futuro e à expectativa do nascimento de um bebê saudável, a termo e bonito, durante a gravidez. As expectativas neutras, por sua vez, referiam-se a características físicas e comportamentais dos bebês, nos relatos das mães MCIE, e a preferência por gênero, nas mães MSIE. Quanto às expectativas negativas, predominou em ambos os grupos o tema sobre o problema da prematuridade do bebê.

Quanto às concepções maternas, predominaram também as concepções positivas, ocorrendo duas vezes mais do que as concepções negativas. Verificou-se que, de forma semelhante em ambos os grupos, predominaram concepções positivas relacionadas ao desenvolvimento, evolução e saúde do bebê e expressões de orgulho e amor pelo bebê. Por outro lado, as concepções negativas das mães dos grupos MCIE e MSIE focalizaram a fragilidade do bebê prematuro. Finalmente, quanto às reações e/ ou sentimentos relatados pelas mães predominaram em ambos os grupos aqueles relacionados às emoções negativas. Os fatores que influenciam na maternidade ocorreram com frequência bem reduzida.

Conforme indicado na Tabela 4 , houve diferença estatisticamente significativa entre os grupos quanto às expectativas maternas neutras e reação e/ou sentimento positivos e negativos. As mães MCIE relataram mais reações e/ou sentimentos, focalizando tanto as emoções positivas quanto

Tabela 1. Características das mães distribuídas em dois grupos diferenciadas quanto à presença ou não de indicadores emocionais de ansiedade e ou depressão materna - Grupo MCIE e Grupo MSIE.

\begin{tabular}{lllc}
\hline Características maternas & $\begin{array}{l}\text { MCIE } \\
(\mathrm{n}=30)\end{array}$ & $\begin{array}{l}\text { MSIE } \\
(\mathrm{n}=30)\end{array}$ & $\begin{array}{c}\text { Valor } \\
\boldsymbol{p} \leq\end{array}$ \\
\hline Idade da mãe $^{1}$ (anos) & $23,7( \pm 6,10)$ & $22,97( \pm 6,96)$ & 0,44 \\
Número de gestações $^{1}$ & $3,21( \pm 2,41)$ & $1,70( \pm 1,09)$ & 0,05 \\
Escolaridade $^{1}$ (anos) & $6,10( \pm 3,18)$ & $7,30( \pm 3,44)$ & 0,19 \\
Ocupação profissional $^{2}$ & & & \\
Empregada $_{\text {Desempregada }}$ & 0,33 & 0,33 & 0,001 \\
$\quad$ Do lar & 0,64 & 0,20 & 0,10 \\
Situação conjugal $^{2}$ & 0,03 & 0,47 & \\
$\quad$ União estável & & 0,72 & \\
Solteira & 0,87 & 0,28 & \\
\hline Nota: MCIE = mães com indicadores emocionais de ansiedade e/ou depressão; MSIE = mães sem indicadores
\end{tabular}

Nota: MCIE = mães com indicadores emocionais de ansiedade e/ou depressão; MSIE = mães sem indicadores emocionais de ansiedade e/ou depressão; ${ }^{1}$ Média e desvio padrão; ${ }^{2}$ Proporção. 
Tabela 2. Características dos neonatos e tempo de internação dos bebês na UTIN, de acordo com sintomas emocionais clínicos maternos de ansiedade e/ ou depressão - Grupo MCIE e Grupo MSIE.

\begin{tabular}{|c|c|c|c|}
\hline $\begin{array}{l}\text { Características dos neonatos e tempo de } \\
\text { internação na UTIN }\end{array}$ & MCIE & MSIE & $\begin{array}{c}\text { Valor } \\
p \leq\end{array}$ \\
\hline Sexo (\% masculino) & 56,66 & 36,66 & 0,26 \\
\hline Idade gestacional ${ }^{1}$ (semanas) & $30,63( \pm 2,33)$ & $31,23( \pm 1,98)$ & 0,22 \\
\hline Peso ao nascimento ${ }^{1}$ (gramas) & $1.079( \pm 260)$ & $1.134( \pm 242)$ & 0,32 \\
\hline Índice de Risco Clínico Neonatal ${ }^{1}$ (CRIB) & $3,59( \pm 3,65)$ & $2,57( \pm 3,12)$ & 0,27 \\
\hline Apgar $5^{\circ}$ minuto ${ }^{1}$ & $8,50( \pm 1,80)$ & $8,80( \pm 1,47)$ & 0,54 \\
\hline \multicolumn{4}{|l|}{ Adequação para a idade gestacional ${ }^{2}$} \\
\hline Pequeno para idade gestacional & 0,80 & 0,73 & 0,77 \\
\hline Adequado para idade gestacional & 0,20 & 0,27 & 0,59 \\
\hline Tempo de internação na UTIN ${ }^{1}$ (dias) & $31( \pm 28)$ & $22( \pm 26)$ & 0,10 \\
\hline
\end{tabular}

Nota: MCIE = mães com indicadores emocionais de ansiedade e/ou depressão; MSIE = mães sem indicadores emocionais de ansiedade e/ou depressão; ${ }^{1}$ Média e desvio padrão; ${ }^{2}$ Proporção.

Tabela 3. Categorias de verbalização referente às concepções maternas acerca do bebê nos grupos MCIE e MSIE - freqüência (f) e porcentagem (\%).

\begin{tabular}{|c|c|c|c|c|}
\hline \multirow[t]{2}{*}{ Categorias e sub-categorias do conteúdo verbal sobre o BEBÊ } & \multicolumn{2}{|c|}{$\begin{array}{l}\text { MCIE } \\
(n=30)\end{array}$} & \multicolumn{2}{|c|}{$\begin{array}{l}\text { MSIE } \\
(n=30)\end{array}$} \\
\hline & f & $\%$ & f & $\%$ \\
\hline EXPECTATIVAS quanto à gravidez/parto/bebê/aleitamento/ maternidade & 148 & 54 & 129 & 57 \\
\hline Positiva & 100 & 68 & 97 & 75 \\
\hline Neutra & 20 & 14 & 24 & 19 \\
\hline Negativa & 17 & 11 & 3 & 2 \\
\hline Conflituosa & 8 & 5 & 5 & 4 \\
\hline Ausência de expectativas & 3 & 2 & 0 & 2 \\
\hline CONCEPÇÕES quanto à gravidez/parto/bebê/aleitamento/ maternidade & 55 & 20 & 51 & 22 \\
\hline Positiva & 34 & 62 & 32 & 63 \\
\hline Negativa & 18 & 31 & 11 & 21 \\
\hline Conflituosa & 2 & 3 & 3 & 6 \\
\hline Neutra & 1 & 2 & 5 & 10 \\
\hline REAÇÃO E/OU SENTIMENTO quanto à gravidez/parto/Bebê/ aleitamento/maternidade/internação & 51 & 18 & 18 & 8 \\
\hline Focalizada na emoção negativa & 33 & 65 & 10 & 56 \\
\hline Focalizada na emoção positiva & 15 & 29 & 6 & 33 \\
\hline Focalizada na emoção conflituosa & 3 & 6 & 2 & 11 \\
\hline DESCRIÇÕES acerca da gravidez/parto/bebê/aleitamento/ maternidade & 8 & 3 & 11 & 5 \\
\hline IDENTIFICAÇÃO DE FATORES QUE INFLUENCIAM NA MATERNIDADE & 2 & 1 & 2 & 1 \\
\hline OUTRAS & 4 & 1 & 6 & 3 \\
\hline RESÍDUO & 8 & 3 & 9 & 4 \\
\hline TOTAL & 272 & 100 & 226 & 100 \\
\hline
\end{tabular}

Nota: MCIE = mães com indicadores emocionais de ansiedade e/ou depressão; MSIE = mães sem indicadores emocionais de ansiedade e/ou depressão. 
as emoções negativas, em comparação com as mães MSIE. Por outro lado, estas expressaram mais expectativas neutras do que as MCIE.

Acrescentando-se à análise específica do grupo MCIE, dividido de acordo com os subgrupos, verificou-se que o MIC (mães com os sintomas emocionais clínicos combinados os tipos ansiedade-estado e ansiedade-traço ou ansiedade e depressão) apresentou significativamente mais expressões sobre reação e/ou sentimento focalizados em emoções negativas do que os demais grupos $[\mathrm{MIC}=20,11( \pm 14,00)$; $\mathrm{MAE}=12,00( \pm 6,26) ; \mathrm{MSCID}=11,43( \pm 12,51) ; \mathrm{MDD}=$ $0( \pm 0) ; p \leq 0,001]$.

\section{Relações entre verbalizações maternas e variáveis maternas e dos bebês}

A Tabela 5 mostra que as concepções positivas maternas acerca do bebê associaram-se significativamente a maior idade gestacional dos bebês, menor tempo de internação na UTIN e menor nível de ansiedade materna do tipo estado. Por outro lado, expectativas negativas e reações e/ou sentimentos negativos relacionaram-se à menor idade gestacional dos bebês. Além disso, as expectativas negativas associaram-se com o menor peso ao nascimento do bebê, enquanto as emoções negativas com maior nível de ansiedade-estado. As reações e/ou sentimentos focalizando emoções conflituosas, por sua vez, associaram-se significativamente com menor índice de depressão materna.

As mães mais jovens apresentaram menos expectativas neutras. Quanto menor o tempo de internação em UTIN e menor a quantidade de intercorrências na evolução clínica do bebê, maior a ocorrência de descrições por parte da mãe sobre os comportamentos do bebê. Deve-se destacar que não houve correlação significativa entre as categorias de conteúdo verbal materno e os eventos vitais potencialmente estressores do ambiente.

\section{Discussão}

Os achados do presente estudo revelaram que as mães de bebês nascidos prematuros, independentemente da presença ou não de indicadores emocionais clínicos de ansiedade e depressão, expressaram, em situação de entrevista guiada, mais verbalizações com conotação positiva do que negativa ao referirem-se aos seus bebês internados na UTIN e no Berçário de Médio Risco. As mães de ambos os grupos, diferenciados pelos sintomas clínicos de ansiedade e depressão, apresentaram expectativas positivas em relação ao desenvolvimento, evolução clínica neonatal e saúde futura do seu bebê. Esses aspectos revelam um lado otimista nas mães que pode atuar como facilitador para o enfrentamento das adversidades decorrentes da prematuridade e do distanciamento do seu bebê devido à internação hospitalar.

Além disso, as mães de ambos os grupos expressaram concepções positivas sobre o bebê. O resultado combinado entre expectativas e concepções positivas reforça a hipótese explicativa de que as mães de neonatos prematuros demonstram alta expectativa em relação ao desenvolvimento de seus bebês e discordam que estes possam vir a ter problemas futuros, como apontado por Pearl e Donahue (1994). A maior frequência de relatos positivos sugere a presença de mecanismos de proteção para o desenvolvimento do bebê, que podem neutralizar efeitos negativos do risco da prematuridade, assim como de recursos emocionais maternos, mesmo em meio a condições adversas.

Por outro lado, em ambos os grupos, as verbalizações maternas com conotação negativa foram relacionadas diretamente às concepções sobre o problema da prematuridade dos bebês. Isso significa que, apesar de ter esperança, as mães experimentam sentimentos negativos relacionados ao nascimento do seu bebê. Como era de se esperar, as mães do presente estudo que apresentaram indicadores emocionais clínicos de ansiedade, associados ou não a sintomas de depressão, relataram mais emoções e sentimentos negativos do que as mães que não apresentavam esses indicadores emocionais.

Verificou-se que $88 \%$ da amostra total das mães não apresentavam antecedentes de história psiquiátrica. Isso indica, portanto, que os indicadores emocionais de ansiedade e depressão estavam relacionados mais à história contemporânea do nascimento do bebê do que à história de saúde mental pregressa materna. Esse dado foi confirmado com a presença marcante da ansiedade do tipo estado medida pelo IDATE. Embora esse tipo de ansiedade tenha característica transitória, deve-se atentar para que os sintomas não sejam intensificados e tornem-se crônicos, afetando negativamente o estado de saúde mental das mães de neonatos prematuros e o cuidado ao bebê.

Tabela 4. Concepções maternas sobre o bebê - categorias com diferenças estatisticamente significativas entre MCIE e MSIE, em termos de média de ocorrência e desvio padrão.

\begin{tabular}{|c|c|c|c|c|c|}
\hline \multirow{2}{*}{ Categorias do conteúdo verbal sobre o bebê } & \multicolumn{2}{|c|}{$\begin{array}{l}\text { MCIE } \\
(n=30)\end{array}$} & \multicolumn{2}{|c|}{$\begin{array}{l}\text { MSIE } \\
(\mathbf{n}=\mathbf{3 0})\end{array}$} & \multirow{2}{*}{ Valor de $p \leq$} \\
\hline & Média & $\begin{array}{l}\text { Desvio } \\
\text { Padrão } \\
\end{array}$ & Média & $\begin{array}{l}\text { Desvio } \\
\text { Padrão } \\
\end{array}$ & \\
\hline Expectativa Neutra & 6,77 & $\pm 10,98$ & 12,50 & $\pm 12,38$ & 0,03 \\
\hline Reação e/ou sentimento focalizado na emoção positiva & 5,43 & $\pm 8,90$ & 2,00 & $\pm 5,66$ & 0,05 \\
\hline Reação e/ou sentimento focalizado na emoção negativa & 12,30 & $\pm 11,94$ & 3,57 & $\pm 6,91$ & 0,01 \\
\hline
\end{tabular}

Nota: MSIE = mães sem indicadores emocionais de ansiedade e/ ou depressão; MCIE = mães com indicadores emocionais de ansiedade e/ou depressão. 
Tabela 5. Coeficientes de correlações de postos de Spearman (r), que foram significativos, entre as categorias verbais sobre o bebê do grupo MCIE e as características maternas, características e evolução clínica dos bebês.

\begin{tabular}{llcc}
\hline Categorias do conteúdo verbal & Variáveis & $\boldsymbol{r}$ & $\boldsymbol{p} \leq$ \\
\hline Concepção Positiva & Idade gestacional $^{1}$ & 0,46 & 0,01 \\
& Tempo de internação em UTIN $^{1}$ & $-0,42$ & 0,01 \\
& Ansiedade-estado $^{2}$ & $-0,50$ & 0,005 \\
Expectativa Negativa & Idade gestacional $^{1}$ & $-0,56$ & 0,001 \\
& Peso ao nascimento $^{1}$ & $-0,38$ & 0,03 \\
Expectativa Neutra & Idade da mãe $^{2}$ & 0,37 & 0,04 \\
Reação e/ ou sentimento focalizando emoções negativas & Idade gestacional $^{1}$ & $-0,40$ & 0,02 \\
& Ansiedade-estado $^{2}$ & 0,43 & 0,01 \\
Reação e/ ou sentimento focalizando emoções conflituosas & Depressão ${ }^{2}$ & $-0,37$ & 0,04 \\
Descrições sobre o comportamento do bebê & Quantidade de intercorrências na $^{2}$ & $-0,44$ & 0,01 \\
& evolução clínica do bebê $^{1}$ & & 0,02 \\
\hline
\end{tabular}

Nota: MCIE = mães com indicadores emocionais de ansiedade e/ ou depressão; ${ }^{1}$ Variáveis dos bebês; ${ }^{2}$ Variáveis maternas.

O indicador de ansiedade materna do tipo situacional relacionou-se com o relato de reações e sentimentos focalizados em emoções negativas. Esse dado sugere que a presença de indicadores de ansiedade tem forte relação com a maneira como as mães reagem ao nascimento do bebê prematuro. Os achados advindos da avaliação psicométrica combinados com os da situação de entrevista podem ajudar no estabelecimento das diretrizes da conduta de intervenção psicológica orientada para as necessidades das mães de forma individualizada, considerando-se o nível dos sintomas emocionais clínicos e os tipos de preocupação.

Vinte mães da amostra do presente estudo (10 com indicadores emocionais clínicos e 10 sem indicadores) foram avaliadas no estudo de Correia \& cols. (2008) em situação de grupo de intervenção psicológica, que ocorreu durante o período de internação do bebê na UTIN ou Berçário de Médio Risco. Os resultados desse estudo mostraram que as mães com indicadores emocionais clínicos continuavam a expressar nos grupos mais sentimentos ou reações com conotação negativa (dúvidas, medo de aproximação do bebê e mal estar, nervosismo, ansiedade ou angústia), em comparação com as mães sem indicadores. As mães de prematuros com índices altos de ansiedade e depressão requerem atenção especial para o adequado enfrentamento do problema da prematuridade e internação do bebê na UTIN. Essas mães devem participar de visitas monitoradas ao bebê no hospital, comunicação com os médicos mediada pelo psicólogo, participação em atendimento psicológico em grupo ou individual, quando necessário, conforme proposto por Linhares e cols. (2006). Após intervenção psicológica envolvendo essas modalidades, durante a hospitalização do bebê na UTIN, mães de bebê pré-termo podem reduzir o nível de ansiedade-estado, como demonstrado por Carvalho, Linhares, Padovani e Martinez (2009).
Interessante observar que, embora haja ansiedade e sentimentos de conotação negativa, as mães do presente estudo com indicadores emocionais clínicos também expressaram significativamente mais reações e sentimentos positivos, relacionados à felicidade de ter o bebê, do que as mães sem os indicadores emocionais clínicos. Esse achado sugere a mescla entre sentimentos negativos e positivos experimentados pelas mães de prematuros, em particular de forma acentuada pelas mães com sintomas clínicos de ansiedade e depressão.

O relato sobre concepções positivas esteve relacionado ao fato de os bebês serem saudáveis e com menor risco neonatal, ou seja, aqueles que tinham maior idade gestacional e menos tempo de internação na UTIN. Por outro lado, quando os bebês apresentavam menor idade gestacional, as mães verbalizaram mais sobre expectativas, reações e sentimentos focalizando emoções negativas. Isso sugere que o bebê mais frágil suscitava mais expectativas e sentimentos negativos nas mães, demonstrando que as condições neonatais e de evolução clínica dos neonatos podiam influenciar a percepção das mães acerca do seu bebê. Consonante com esse resultado, o menor peso ao nascimento do bebê também esteve relacionado à maior expressão de expectativas negativas por parte das mães.

Verificou-se, no presente estudo, a existência de relações entre menor tempo de internação do bebê na UTIN, menor quantidade de intercorrências na sua evolução clínica e mais relatos por parte das mães envolvendo descrições sobre seu comportamento. Isso pode se dar pelo fato de as mães de bebês com menor risco e boa evolução conseguirem ter maior contato e aproximação com os mesmos. Por outro lado, quando o bebê está clinicamente menos estável e mais grave, muitas vezes a mãe pode ter maior restrição de contato com seu bebê ou distanciar-se deste a fim de proceder à autoproteção emocional. Segundo Kennell e Klaus (1998), o 
comportamento de distanciamento do bebê pode representar um "luto antecipatório", ou seja, uma preparação por parte dos pais para possível óbito do bebê. A situação é altamente ansiogênica, o bebê responde pouco e a mãe acaba afastandose para não sofrer; diante disso, há uma restrição de oportunidades de observar, conhecer e vincular-se a ele.

Em suma, o presente estudo mostrou que a entrevista guiada é sensível para avaliar concepções, sentimentos, reações e expectativas, relatadas por mães de bebês prematuros, revelando a presença de expectativas e concepções positivas e sentimentos negativos em ambos os grupos. Houve associação entre a presença de indicadores emocionais clínicos de ansiedade e depressão materna, maior risco e gravidade clínica dos bebês e sentimentos e reações negativos relatados pelas mães. A combinação entre as medidas psicométricas do estado emocional materno e a entrevista guiada para avaliar concepções, sentimentos e expectativas maternas mostrou ser um procedimento de avaliação útil a fim de dimensionar as dificuldades e os recursos psicológicos das mães de bebês nascidos prematuros e hospitalizados em UTIN e Berçários de Médio Risco.

Alguns desdobramentos do presente estudo podem ser apontados. Primeiramente, pode ser ampliada a compreensão para outros aspectos relativos à experiência materna com o nascimento prematuro do bebê, tais como os cuidados maternos e amamentação. Em segundo lugar, pode se realizar estudos comparando as concepções das mães de bebês prematuros com mães de bebês a termo, a fim de analisar a especificidade das concepções e sentimentos de mães de bebês prematuros.

\section{Referências}

Andrade, L., \& Gorenstein, C. (2000) Aspectos gerais de escalas de avaliação de ansiedade. Em C. Gorenstein, L. H. S. G. Andrade \& A. W. Zuardi (Orgs.), Escalas de avaliação clinica em Psiquiatria e Psicofarmacologia (pp. 139-144). São Paulo: Lemos.

Aylward, G. P. (2002). Methodological issues in outcomes studies of at-risk infants. Journal of Pediatric Psychology, 27, 37-45.

Biaggio, A., \& Natalício, L. (1979). Inventário de Ansiedade TraçoEstado. Rio de Janeiro: CEPA.

Carvalho, A. E. V. (2005). Indicadores emocionais maternos $e$ intervenção psicológica durante hospitalização do bebê prétermo em UTI Neonatal. Tese de Doutorado, Universidade de São Paulo, Ribeirão Preto.

Carvalho, A. E. V., Linhares, M. B. M., Padovani, F. H. P., \& Martinez, F. E. (2009). Anxiety and depression in mothers of preterm infants under psychological intervention during NICU hospitalization. The Spanish Journal of Psychology, 12, 161-170.

Cockburn, F., Cooke, R. W. I., Gamsu, H. R., Geenough, A., Hopkins, A., Mcintosh, N., Ogstont, S. A., Parryt, G. J., Silverman, M., Shaw, J. C. L., Tarnow-Mordit, W. O., \& Wilkinson, A. R. (1993). The CRIB (Clinical Risk Index for Babies) score: A tool for assessing initial neonatal risk and comparing performance of Neonatal Intensive Care Units. Lancet, 342, 193-198.
Correia, L. L., Carvalho, A. E. V., \& Linhares, M. B. M. (2008). Conteúdos verbais expressos por mães de bebês prematuros com sintomas emocionais clínicos. Revista Latino- Americana de Enfermagem, 16, 64-70.

Correia, L. L., \& Linhares, M. B. M. (2007). Ansiedade materna nos período pré- e pós- natal: revisão da literatura. Revista Latino-Americana de Enfermagem, 15, 677-683.

Cunha, J. A. (2001). Manual da versão em português das Escalas Beck. São Paulo: Casa do Psicólogo.

Davis, L., Edwards, H., Mohay, H., \& Wollin, J. (2003). The impact of very premature birth on the psychological health of mothers. Early Human Development, 73, 61-70.

Del Ben, C. M. (1995). Estudo de confiabilidade do diagnóstico psiquiátrico obtido através da Entrevista Clínica Estruturada do DSM III-R (SCID) em serviço ambulatorial de um hospital escola. Tese de Doutorado, Universidade de São Paulo, Ribeirão Preto.

Doering, L. V., Moser D. K., \& Dracup, K. (2000). Correlates of anxiety, hostility, depression, and psychosocial adjustment in parents of NICU infants. Neonatal Network, 19, 15-23.

Feldman, R. (2007). Maternal versus child risk and the development of parent-child family relationships in five-risk populations. Development and Psycopathology, 19, 293-312.

Flacking, R., Ewald, U., \& Starrin, B. (2007). "I wanted to do a good". Experiences of 'becoming a mother' and breastfeeding in mothers of very preterm infants after discharge from Neonatal Unit. Social Science \& Medicine, 64, 2405-2416.

Glaser. A., Bucher, H. U., Moergeli, H., Fauchère, J. C., \& Buechi, S. (2007). Loss of preterm infant: Psychological aspects in parents. Swiss Medical Weekly, 137, 392-401.

Gorenstein, C., \& Andrade, L. (2000). Inventário de Depressão de Beck - propriedades psicométricas da versão em português. Em C. Gorenstein, L. H. S. G. Andrade \& A. W. Zuardi, (Orgs.), Escalas de avaliação clinica em Psiquiatria e Psicofarmacologia (pp. 89-95). São Paulo: Lemos.

Javorski, M., Caetano, L. C., Vasconcelos, M. G. L., Leite, A. M., \& Scochi, C. G. M. (2004) As representações sociais do aleitamento materno para mães de prematuros em Unidade de Cuidado Cangurú. Revista Latino-Americana de Enfermagem, 12, 890-898.

Kennell, J. H., \& Klaus, M. H. (1998). Bonding: Recent observations that alter perinatal care. Pediatrics in Review, 19, 4-12.

Klaus, M. H., \& Kennell, J. H. (1993). Atendimento para os pais de bebês prematuros ou doentes. Em M. H. Klaus \& J. H. Kennell (Orgs.), Pais-bebê: a formação do apego (pp. 170-244) (D. Batista, Trad.). Porto Alegre: Artes Médicas. (Trbalho original publicado em 1992).

Linhares, M. B. M., Carvalho, A. E. V., Correia, L.L., Gaspardo, C. M., \& Padovani, F. H. P. (2006). Psicologia Pediátrica e Neonatologia de alto risco: promoção precoce do desenvolvimento de bebês prematuros. Em M. A. Crepaldi, M. B. M Linhares \& G. B. Perosa (Orgs.), Temas em Psicologia Pediátrica (pp. 109-145). São Paulo: Casa do Psicólogo.

Linhares, M. B. M., Carvalho, A. E. V., Padovani, F. H. P. Bordin, M. B. M., Martins, I. B., \& Martinez, F. E. (2004). A compreensão do fator de risco da prematuridade sob a ótica desenvolvimental. Em E. M. Marturano, M. B. M. Linhares \& S. M. Loureiro (Orgs.), Vulnerabilidade e proteção: indicadores na trajetória de desenvolvimento do escolar (pp.11-38). São Paulo: Casa do Psicólogo/ FAPESP. 
Marques, C. S. B. (2003). Classificação do recém-nascido segundo idade gestacional e crescimento fetal. Em P. Pachi (Org.), $O$ pré-termo: morbidade, diagnóstico e tratamento (pp. 23-40). São Paulo: Roca.

Monteiro, T. M. T., Silva, L. M. S., \& Silva, M. V. S. (2002). Reações de mães diante do nascimento de um filho prematuro. Cogitare Enfermagem, (Curitiba), 7, 36-42.

Padovani, F. H. P (2005). Indicadores emocionais de ansiedade, disforia e depressão e verbalizações maternas acerca do bebê, da amamentação e da maternidade em mães de bebês nascidos pré-termo de muito baixo peso, durante a hospitalização do bebê e após a alta, comparada a mães de bebês nascidos a termo. Tese de Doutorado, Universidade de São Paulo, Ribeirão Preto.

Padovani, F. H. P., Linhares, M. B. M., Carvalho, A. E.V., Duarte, G., \& Martinez, F.E. (2004). Avaliação de sintomas de ansiedade e depressão em mães de neonatos pré-termo durante e após hospitalização em UTI-Neonatal. Revista Brasileira de Psiquiatria. (São Paulo), 26, 251-254.

Pearl, R., \& Donahue, M. (1994). Brief report: Four years after a preterm birth: Children's development and their mothers' beliefs and expectations. Journal of Pediatric Psychology, 20, 363-370.

Rice, F., Jones, I., \& Thapar, A. (2007). The impact of gestational stress and prenatal growth on emotional problems in offspring: A review. Acta Psychiatry Scandivava, 115, 151-183.
Roller, C. G. (2005). Getting to know you: Mother's experiences of Kangaroo Care. Journal of Obstetrics and Gynecology Neonatal Nursing, 34, 210-217.

Savoia, M. G. (2000). Instrumentos para avaliação de eventos vitais e de estratégias de enfrentamento (coping) em situação de estresse. Em C. Gorenstein, L. H. S. G. Andrade \& A. W. Zuardi (Orgs.), Escalas de avaliação clínica em Psiquiatria e Psicofarmacologia (pp. 377-383). São Paulo: Lemos.

Spitzer, R. L., Williams, J. R., Gibbon, M., \& First, M. B. (1989) Instruction manual for the structured clinical interview for DSM III-R. New York: State Psychiatric Institute.

Zanardo, V., \& Freato F. (2001). Home oxygen therapy in infants with bronchopulmonary dysplasia: Assessment of parental anxiety. Early Human Development, 65, 39-46.
Recebido em 03.01.07

Primeira decisão editorial em 27.03.08

Versão final em 18.04.08

Aceito em 20.06.08 